\title{
Android y Smartphones: una computadora de bolsillo
}

Br. Elmer Miroslav Mosher Golovin.

Facultad Regional Multidisciplinaria Matagalpa. ${ }^{1}$

Recibido: 24 de abril 2013- Aprobado: 12 noviembre 2013.

\section{RESUMEN}

Este ensayo describe las oportunidades y posibilidades que ofrecen al usuario los Smartphones y el Sistema Operativo (SO) Android. Se explica el concepto de Android y Android Open Source Project (AOSP), además de los dispositivos que soportan Android y las aplicaciones Google experience. También, los conceptos del PC portátil y el Smartphone, comparando sus propósitos y funciones principales. Se relata sobre cómo Ubuntu puede ser utilizado en Android. De igual forma se narra sobre las aplicaciones nativas de Android y su potencial. También se aborda sobre las modificaciones para Android y lo que ofrecen y se explica de manera breve como sacarle partido al dispositivo Android. Para realizar este trabajo se hizo la revisión de bibliografía actualizada y se realizaron pruebas prácticas tanto con los Smartphones y el SO Android, como con las computadoras portátiles para cerciorarse de la veracidad de la información teórica encontrada, poniéndola en práctica. Se concluye que la combinación de estos dos, Smartphones y Android, genera un dispositivo móvil, versátil y de fácil uso, lo que permite llevar el trabajo y entretenimiento a todas partes, y lo más importante, sin perder lo que se puede hacer en un sistema estacionario. Así, el PC portátil es rediseñado a un tamaño micro, y con características técnicas muy buenas, logrando un auténtico "PC de bolsillo".

Palabras claves: android, usuario, Smartphone, PC portátil.

\section{INTRODUCCIÓN}

En la actualidad el computador portátil y el concepto de movilidad se ha ido "fundiendo" con nuestras vidas cotidianas y labores, tanto así que es más productivo y atractivo el individuo que posea y utilice tecnología móvil que el que no. Este trabajo trata sobre las capacidades de los SO móviles actuales para sernos de utilidad, en particular, el SO Android. Es un ensayo de carácter práctico-informativo para que el público que no conozca mucho sobre la temática pueda informarse y tener un mayor conocimiento para su propio beneficio. El presente trabajo se ha realizado con el propósito de demostrar las capacidades y oportunidades que presentan los Smartphones y la plataforma móvil Android. Este ensayo se destina al público con conocimientos informáticos medios en tecnicismos, para que se instruyan aún más y puedan aprovecharse de la información de este documento.

\section{DESARROLLO}

\section{¿Qué es Android?}

De acuerdo a (Google, 2012), Android es la plataforma móvil más popular del globo, que incluye las aplicaciones Google como Gmail, Google Search, Google Voice, entre otros. Android nace alrededor del

\footnotetext{
${ }^{1}$ Trabajo dirigido por la maestra Natalia Sergueyevna Golovina
} 
año 2007 como parte de un grupo desarrollador independiente, en el 2008 es adquirido por la Open Handset Alliance, con Google como principal promotor. Android ofrece cientos de miles de aplicaciones en su Play Store (también conocido como Google Play y AndroidMarket en versiones anteriores), además de millones de músicas, videos y libros a través de aplicaciones especializadas.

\section{Dispositivos Android}

Según (Ziegler, 2011), aquel dispositivo que incluya un sistema operativo realizado con el código del AOSP, además de contener las aplicaciones Google de fábrica es considerado un dispositivo Android. El dispositivo Android también se caracteriza por brindar la "experiencia Google", que consiste de las aplicaciones de código cerrado propias de Google, así como la Play Store y Google Voice.

\section{Aplicaciones Google experience y AOSP}

Acorde a (Google, 2011), las aplicaciones Google experience están conformadas un conjunto relativamente pequeño de servicios ofrecidos solamente por Google, más sin embargo, de gran utilidad. Entre las más destacadas de estas aplicaciones está Google Search, el buscador de Google especialmente diseñado para utilizarse en dispositivos Android; Google Maps, mapas del globo con infinidad de detalles; Gmail, el correo electrónico de Google; YouTube, el gestor para ver videos online; y Play Store, la tienda virtual de Google para la venta e instalación de aplicaciones de todo tipo. "El Android Open Source Project, encabezado por Google, está programado para el mantenimiento y futuro desarrollo de Android." (Google, 2012). Así, este proyecto de constante desarrollo y de código abierto apoyado por decenas de compañías líderes en ordenadores y electrónica, es el responsable de mantener actualizados y agregar cada vez más funciones atractivas a los dispositivos Android, y siempre, de manera gratuita.

\section{El PC portátil y el Smartphone}

Callejas (2012) menciona que lo principal en términos de lo que puede hacer un PC, un Smartphone y un Tablet. Básicamente, todos útiles, y en ciertos escenarios necesarios, pero ninguno más de ayuda que el PC y el Smartphone. Cada vez cada uno obtiene una interfaz de usuario más parecida al del otro, lo que facilita al usuario pasarse de trabajar de un dispositivo a otro sin sentirse en otro ambiente o con dificultades mayores de uso, lo cual resulta en algo cómodo y atractivo, y en particular, con Android que se centra cada vez más en la facilidad de uso y funcionalidad.

El potencial de un PC ha crecido enormemente en los últimos años, e igual ha sido el caso con los Smartphones. Ahora con procesadores nVidiaTegra 3 de cuatro núcleos a $1.5 \mathrm{GHz}$ y unos gráficos tan buenos como para jugar en tres dimensiones con emulaciones de la física e iluminación asombrosas. Esto ha permitido a los Smartphones abrirse paso en qué aplicaciones pueden abrir, y para que lo podemos utilizar, inclusive, AutoCAD ya es un producto disponible para Android desde el año 2011, tanto restringido claro, pero para ser móvil hace más que suficiente.

\section{Ubuntu y Android}

Ahora los dispositivos Androidmulti-núcleo pueden ser PCs también. Ubuntu para Android permite a dispositivos Android de gama alta ejecutar Ubuntu, el sistema operativo gratuito de escritorio favorito del globo. Así los usuarios obtienen el Android que conocen en movilidad, pero cuando conectan su Smartphone a un monitor, mouse y teclado, se convierte en un PC. (Canonical, 2012).

Linux es un SO ya de años y años de evolución, es robusto y gratuito, y es soportado en tantas arquitecturas. Android puede ser ejecutado en arquitecturas x86 (32 bits) como las del PC ordinario, y en arquitecturas ARM (Advanced RISC Machines) como las del Smartphone. Ubuntu, una de las tantas 
distribuciones de Linux, tiene ya soporte para ARM, además del soporte x86 y x64 que ofrece desde sus inicios, y no se ha quedado corto con su distribución ARM, literalmente, cualquier dispositivo Android con medio giga en RAM, un CPU de un GHz o más y acceso root (también conocido como SU o SuperUser) es capaz de ejecutar las distribuciones ARM de Ubuntu con la ayuda de una ISO descargada y algunos comandos ejecutados en la terminal de Linux. Esto abre un camino enorme a lo que se puede hacer en el Smartphone, podemos abrir LibreOffice, o algún conversor PDF, y porque no un navegador web con las prestaciones de un navegador de escritorio como FireFox o Chrome, y tampoco olvidarse de algún entorno de desarrollo en Java, Ruby, .NET, entre otros. Por ahora, ARM es un sector creciente, Windows 8 ya tiene su distribución ARM (Windows RT) a ser utilizada en tablets, y el software x86-64 no funciona con un simple doble click en ARM, éste debe ser recompilado en ARM con ayuda del código fuente para que las instrucciones de la aplicación puedan ser interpretados correctamente por un procesador ARM.

\section{Aplicaciones nativas de Android y su potencial}

"Ahora puedes llevar tu trabajo a donde sea con la ayuda de DocumentsToGo para ver, editar y crear archivos de Microsoft Word, Excel and PowerPoint así como ver archivos de Adobe PDF en tu Smartphone Android. Y debido a que tiene la totalidad de funciones y fácil de usar, DocumentsToGo es una de las suites móviles de oficina que da sentido al buen negocio." (DataViz, 2012). Y así existen diversidad de aplicaciones nativas de Android que nos ayudan en prácticamente todo, incluso, hay cuales compiten entre su misma categoría como QuickOffice para ofimática y DocumentsToGo para la misma categoría. En la Play Store de Google hay miles de aplicaciones de productividad, se puede editar los archivos de audio, hacer mezclas musicales, o pulir una diapositiva de PowerPoint antes de exponerla. Y más centrados en lo que se hace en el ordenador, pues se puede comprar un teclado USB o Bluetooth, conectarlo al Smartphone mediante USB OnTheGo(OTG) o Bluetooth y si no basta la pantalla pues conectarle una mediante VNC o HDMI, y finalmente poner a reproducir la música y comenzar a trabajar en Office, Dibujo, o lo que se necesite, y si senecesita información de una memoria USB pues también se conecta mediante USB OTG.

USB OTG es un módulo exclusivo en el kernel Linux, que permite manejar un dispositivo USB como Host y utilizarlo como lo utilizamos en el PC. Funciona para PenDrives, cámaras, teclados, mouse, entre otros. Para usarlo en Android se requiere que el kernel que se utilice soporte USB OTG, además del puerto USB en el Smpartphone o Tablet y la debida alimentación eléctrica. No es un estándar, pero es instalable con la ayuda de un poco de tiempo, paciencia y documentación adecuada sobre cómo añadir módulos al kernel, incluso, hay páginas web sobre tutoriales paso a paso y kernels ya modificados para obtener USB OTG en el Smartphone.

\section{Modificaciones para Android y lo que ofrecen}

"CyanogenMod es un firmware post-compra para un numero de Smartphones basados en el sistema operativo de código abierto Android. Éste ofrece funciones no encontradas en el firmware oficial de Android de los vendedores de estos Smartphones." (CyanogenMod, 2012).

Cada dispositivo Android tiene sus "exclusivas" acorde la compañía telefónica que lo patrocina, teniendo unos más atractivos y funciones que otros. CyanogenMod extingue estas diferencias para los dispositivos que soporta, ofreciendo aún más atractivos y funciones que los dispositivos populares como la serie Galaxy de Samsung, con mejor rendimiento y actualizaciones más constantes.

Hay una gama de dispositivos muy especiales en lo que respecta a Android, y son tan especiales debido a que son diseñados por la misma Google y tienen literalmente las más constates actualizaciones y mejor 
soporte para funciones nuevas. Estos dispositivos son la línea Nexus, que comenzó con el NexusOne, le sigue el Nexus S, luego el GalaxyNexus, después el Nexus 7 y finalmente el Nexus Q. Lo especial de la línea Nexus es que hay código fuente para sus drivers y soporte especial y oficial por parte de Google para desarrollo en estos dispositivos, haciendo más fácil y rápida la creación de nuevas aplicaciones y proyectos para los Nexus como drivers para mejores gráficos, soporte de más dispositivos Bluetooth, inclusión de nuevas funciones y características, entre otros. Así hay también dispositivos Android como el Motorola DROID 2 Global que son todo lo contrario de la línea Nexus, son bloqueados por todas partes, ningún soporte para kernels no oficiales u otras funciones, ningún código fuente de drivers, etc. Es por esto que hay dispositivos con mejor y peor soporte de modificaciones tanto por parte de CyanogenMod como de desarrolladores independientes, y no es propiamente por culpa del dispositivo, si no de los creadores del mismo y la poca libertad que ofrecen.

Otra modificación muy llamativa es el "rooteo", que es la obtención del acceso de la cuenta SU de Linux (recordemos que Android es una distribución de Linux), permitiéndonos una innumerable cantidad de extras, como compartir nuestro internet móvil mediante WiFi, Bluetooth o USB, modificar detalles de la interfaz de usuario, utilizar Ubuntu para Android, instalar un kernel con soporte para USB OTG, instalar CyanogenMod en el mismo, instalar BusyBox (conjunto de comandos de la terminal de Linux) y realizar tareas como ping, tracert, sripting, entre otros. Es apreciable que hay dos cosas principales que se obtienen al modificar el dispositivo Android, librarse de las restricciones impuestas por el fabricante y la compañía telefónica, y obtener innumerable cantidad de funciones para explotar al máximo el Smartphone.

\section{Como sacarle partido al dispositivo Android}

"Los teléfonos Android han crecido en popularidad debido en gran parte a la evolución de sus características. En ocasiones, es fácil comenzar a usar este tipo de teléfonos, pero en otras puede ser un poco intimidante aprovechar todas las capacidades de la plataforma por parte de los nuevos usuarios." (Caraballo, 2010). El ver y escuchar cosas como rootear, cambiar kernel, cambiar ROM, Live OC, etc. es confuso para muchos usuarios de dispositivos Android, aún más para los usuarios que son "nuevos". Android tiene un potencial enorme, pero éste depende de que tanto partido le saque el usuario. Es cierto que un dispositivo Android tiene muy poco que envidiarle a un PC estándar, pero, ¿qué tal si los usuarios ni siquiera saben que esto es posible y solo lo utilizan para llamar y mensajería?

Pues ahí el gran dilema, y en la gran mayoría de plataformas sucede, quien no dice que es mejor Windows que Linux porque es más fácil de usar, y quien no que Linux es mejor que Windows por tener más funciones y ser gratuito. Android no es la excepción, hay muchos que lo critican, y otros que lo favorecen, ya sea respecto a MeeGo, iOS o BlackBerry OS. Bueno, pero ¿cómo hacerle para sacarle provecho al dispositivo Android, así como se lo saca al PC? Pues la solución es sencilla pero de esfuerzo requiere, y eso es lo que a la mayoría de usuarios no les agrada. Por ejemplo, con tan solo invertir un par de horas en Ubuntu para Android y rooteo y descargar unos $700 \mathrm{MiB}$, ya se tendrá un dispositivo Android corriendo Ubuntu y haciendo maravillas. Sacarle partido al dispositivo Android consiste básicamente en esforzarse en indagar y descubrir, además de ser travieso sin dejar en el olvido el ser cauteloso. No es sólo una la característica de Android que no está bien documentada y es extremadamente útil (caso del tethering/compartir internet por ejemplo), y aprenderlas no es difícil, pero si requiere paciencia y un tanto de tiempo. En esto puede ser aprovechado el tiempo libre que se tiene y tomarlo a modo de pasatiempo y así poco a poco lograr instalar y configurar las aplicaciones que realicen lo mismo que se hace en el PC y lograr así un "PC de bolsillo".

\section{CONCLUSIÓN}


El Smartphone es un dispositivo que apareció alrededor del 2008 para el público en general, y de muchas maneras sigue siendo un misterio para muchos el que hace y que lo hace diferente. Con la aparición del Smartphone aparecen también los SO móviles más completos, como Android, que brindan un aprovechamiento tan exquisito al Smartphone que permite realizar prácticamente todo lo que hace una PC de escritorio o portátil. La combinación de estos dos, Smartphones y Android, genera un dispositivo móvil, versátil y de fácil uso, lo que permite llevar el trabajo y entretenimiento a todas partes, y lo más importante, sin perder lo que se puede hacer en un sistema estacionario. Así, el PC portátil es rediseñado a un tamaño micro, y con características técnicas muy buenas, logrando un auténtico "PC de bolsillo".

\section{BIBLIOGRAFÍA}

Callejas, I. (3 de 4 de 2012). El androide libre. Recuperado el 1 de 5 de 2012, de Mi escritorio de Smartphone, tablet y PC: Cada vez más unidos y parecidos: http://www.elandroidelibre.com/2012/04/mi-escritorio-de-smartphone-tablet-y-pc-cada-vez-masunidos-y-parecidos.html

Canonical. (2012). Ubuntu. Recuperado el 30 de 6 de 2012, de Ubuntu for Android: http://www.ubuntu.com/devices/android

Caraballo, Y. (14 de 10 de 2010). Movil Arena. Recuperado el 29 de 7 de 2012, de 5 consejos para aprovechar al máximo tu teléfono Android: http://movilarena.com/2010/10/14/5-consejos-paraaprovechar-al-maximo-tu-telefono-android/

CyanogenMod. (2012). CyanogenMod. Recuperado el 8 de 25 de 2012, de CyanogenMod - Homepage: http://www.cyanogenmod.com/

DataViz. (2012). DataViz. Recuperado el 5 de 5 de 2012, de Documents To Go for Android Smartphones: Word and Excel on your Android Smartphone: http://www.dataviz.com/products/documentstogo/android/

Google. (2011). Google Móviles. Recuperado el 27 de 7 de 2012, de Google para Android: http://www.google.com/mobile/android/

Google. (2012). Android. Recuperado el 05 de 02 de 2012, de Android - Discover Android: http://www.android.com/about/

Google. (2012). Android Open Source Project. Recuperado el 17 de 4 de 2012, de About the Android Open Source Project: http://source.android.com/about/index.html

Ziegler, C. (29 de 12 de 2011). The Verge. Recuperado el 13 de 6 de 2012, de What is an 'Android device'?: http://www.theverge.com/2011/12/29/2668214/what-is-an-android-device 